\title{
The multifaceted contributions of long noncoding RNAs on mitochondrial dysfunction in diabetic nephropathy
}

Received 19 December 2020

Accepted 20 January 2021

$\mathrm{Li} \mathrm{Li}^{1,2}$, Lin Sun², Farhad R. Danesh ${ }^{1,3, *}$

'Section of Nephrology, Division of Internal Medicine, The University of Texas at MD Anderson Cancer Center, Houston, TX, USA

${ }^{2}$ Department of Nephrology, The Second Xiangya Hospital, Central South University, Changsha, Hunan Province, China

${ }^{3}$ Department of Pharmacology \& Chemical Biology, Baylor College of Medicine, Houston, TX, USA

Diabetic kidney disease (DKD) is the leading cause of chronic kidney disease (CKD) and end-stage renal disease worldwide [1]. Consensus guidelines for the management of CKD in patients with diabetes recommend control of hyperglycemia and hypertension as well as the use of renin-angiotensin system blockers, and, more recently, sodium-glucose cotransporter 2 inhibitor [2, 3]. Nonetheless, despite extensive studies on the pathogenesis and development of DKD over the past three decades, a significant risk of DKD progression remains, and hence novel approaches and therapies are needed to prevent its occurrence.

Long noncoding RNAs (IncRNAs) are the largest and most diverse class of noncoding RNAs that are classically defined as having >200 nt in length, transcribed by RNA polymerase II, capped, spliced, and polyadenylated but classically lack a significant open reading frame [4]. It is now widely accepted that IncRNAs have diverse regulatory roles in gene expression and are involved in transcriptional regulation, molecular scaffolding, decoys for other RNAs or proteins, and chromosome remodeling [4]. However, while thousands of IncRNA transcripts have been cataloged, the biological function of the majority of them still remains poorly understood. The function of several IncRNAs is just a beginning to be unraveled in various kidney diseases, including in DKD.

Among different IncRNAs screened for their contributions to the development and progression of DKD, IncRNA Erbb4-IR was initially reported to be involved in the pathogenesis of DKD through a TGF- $\beta /$ Smad3-dependent pathway [5]. It was shown that Erbb4-IR knockdown protected against proteinuria and kidney fibrosis in $d b / d b$ diabetic mice, an established murine model of type 2 diabetes. GAS5 is another IncRNA that was reported to play a role in the progression of DKD [6]. GAS5 seems to act as a miRNA sponge to protect the kidney against mesangial proliferation and fibrosis [7]. Kato et al [8]. more recently identified the host IncRNA of a megacluster of nearly 40 miRNAs that play a key role in the development of DKD. The authors convincingly showed that the expression of IncRNA IncMGC was upregulated under high glucose condition or TGF- $\beta 1$ stimulation [8]. IncRNA metastasis-associated lung adenocarcinoma transcript 1 (MALAT1) expression was also shown to be associated with podocyte apoptosis that is induced by high glucose [9].

One central but understudied feature of IncRNAs is that the specific subcellular distributions of IncRNAs are critical for their function. Precisely, the subcellular distribution of IncRNAs in the mitochondria has been gaining attention as direct or indirect regulators of the mitochondrial function. Until now, >20 IncRNAs that could affect mitochondrial biology directly or indirectly have been described. These IncRNAs impact various mitochondrial functions by directly targeting or indirectly influencing mitochondrial-related genes and proteins, and, therefore, these IncRNAs could present possible novel treatment strategies.

In general, there are two types of IncRNAs that could impact mitochondrial function (Figure 1). The first one is represented by the nuclear-encoded IncRNAs. Several nuclear-coded IncRNAs have been shown to modulate mitochondrial function indirectly. For instance, we have recently shown that IncRNA taurine upregulated gene 1 (Tug1) regulates mitochondrial function in podocytes by targeting the expression of PGC-1 $\alpha$ [10]. Tug1-binding site was identified upstream of the Ppargc1a promoter region. Tug1's interaction with this region recruited Pgc-1 $\alpha$ to promote its own gene transcription. Tug1 exhibits a renoprotective phenotype in DKD by rescuing the expression of PGC-1 $\alpha$ and improving mitochondrial bioenergetics under high glucose conditions. Podocyte-specific transgenic expression of Tug1 in diabetic mice $(d b / d b)$ improved glomerular basement membrane (GBM) thickening and reduced podocyte apoptosis, leading to amelioration of albuminuria and DN progression [10]. In patients with DN, lower levels of Tug1 expression were correlated with reduced levels of estimated glomerular filtration rate (eGFR)[10]. Another example of nuclear IncRNA affecting mitochondrial function in podocytes in the diabetic milieu is maternally expressed gene 3 (Meg3) [11]. Excessive mitochondrial fission of podocytes and renal histopathology were improved in podocyte-specific 


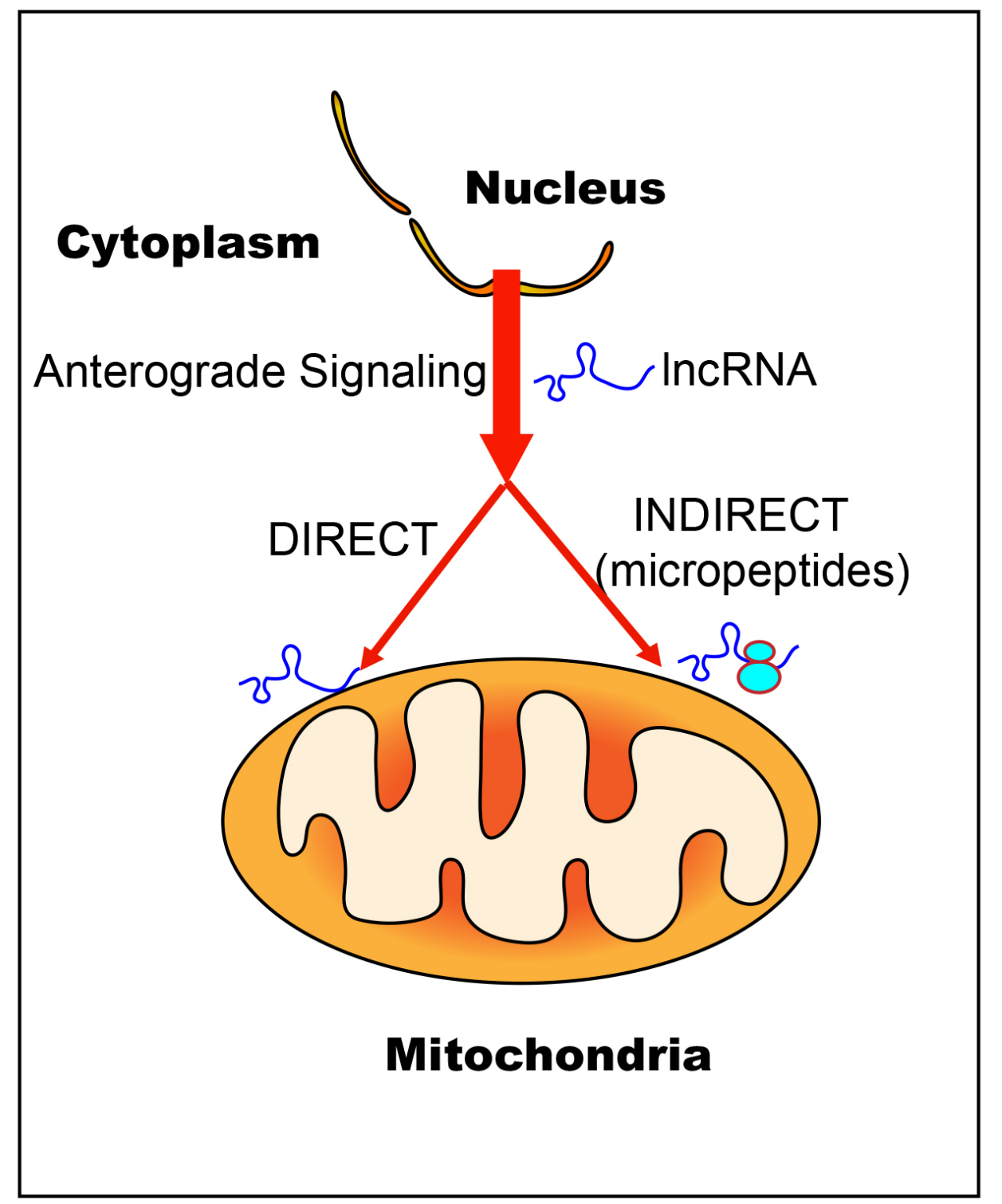

Figure 1. Multifaceted effects of IncRNA on mitochondrial function.

Meg3 knockdown diabetic mice. Meg3 knockout podocytes exhibited elongated mitochondria with attenuated podocyte damage as well as decreased mitochondrial translocation of dynamin-related protein 1 (Drp1). Limited nuclear-encoded IncRNAs are found to be residing in mitochondria, referred to as nuclear-transported mitochondria-associated IncRNAs (ntmtlncRNAs) [12]. One example is IncRNA SAMMSON, which was indicated to be localized to mitochondria by RNA FISH and cell fraction experiments. Its knockdown resulted in mitochondria synthesis defects and reduced OXPHOS complex I and IV activities [13]. However, it remains to be deciphered how they can be translocated to and inside the mitochondria and whether those IncRNAs play a role in DN.

The second category of IncRNAs that could affect mitochondrial function is mitochondrial-encoded IncRNAs (mt-IncRNAs). The first evidence of human mitochondrial IncRNAs was described in 2011 [14]. The authors observed that $15 \%$ of the mitochondrial transcriptome (after removing rRNA and tRNA) were represented by ncRNAs. Mt-IncRNAs could be broadly classified into simple antisense mt-IncRNAs and chimeric mt-IncRNAs since some of the mt-IncRNAs are chimeric, which means that they are derived from more than one gene that ultimately merges their transcripts into one [15]. Mt-IncRNAs are found to be retrograde signals that report the mitochondrial activity to the nucleus [16]. However, the physiological functions of mt-IncRNAs, in general, are not yet well-understood, and their contributions in DKD are largely unexplored.

An interesting recent discovery in the field of IncRNAs has been that some IncRNAs can be protein coding and contain short open reading frames (sORFs) that have been 
overlooked because of their small size. Many of these sORFs encode micropeptides or small proteins with fundamental biological importance, including their effects on mitochondrial biology. As a good example, two independent groups have recently reported a mitochondrial peptide encoded by LINC00116 that could enhance mitochondrial respiratory efficiency $[17,18]$. Tug1 gene locus also contains at least one ORF that produces a microprotein that localizes to mitochondria. Ectopic expression of this microprotein was shown to affect mitochondria membrane potential [19]. Considering the fact that mitochondrial proteome is enriched in micropeptides, accounting for $5 \%$ of its proteins, [20] micropeptides encoded by IncRNAs remains an intriguing and largely unexplored field in mitochondrial biology. It is important to delve deeper into the functional analysis of these micropeptides and identify whether the protective effects of Tug1 on the progression of DKD are, at least in part, dependent on these micropeptides.

\section{Acknowledgment}

We thank all members of Danesh's laboratory for their helpful discussions, including Drs. Benny Chang, Daniel Galvan, Koki Mise, and Jianyin Long.

Conflict of Interest

The authors declare that they have no conflicts of interest. Farhad R. Danesh is the co-editor in chief of the journal.

\section{Funding Sources}

This work was supported by grants from the National Institutes of Health RO1DK078900 (F.R.D) and R01DK091310 (F.R.D).

Author Contributions

L.L. and F.R.D. contributed to the writing of this manuscript. All authors have read and approved the final manuscript.

\section{REFERENCES}

[1] United States Renal Data System. 2019 USRDS annual data report: Epidemiology of kidney disease in the United States. National Institutes of Health, National Institute of Diabetes and Digestive and Kidney Diseases, Bethesda, MD, 2019.

[2] KDOQI Clinical Practice Guidelines and Clinical Practice Recommendations for Diabetes and Chronic Kidney Disease. Am J Kidney Dis 2007; 49:S12-154.

[3] Marx N, Davies MJ, Grant PJ, Mathieu C, Petrie JR, Cosentino $\mathrm{F}$, et al. Guideline recommendations and the positioning of newer drugs in type 2 diabetes care. Lancet Diabetes Endocrinol 2020; 9:46-52.

[4] Yao RW, Wang Y, Chen LL. Cellular functions of long noncoding RNAs. Nat Cell Biol 2019; 21:542-51.

[5] Sun SF, Tang PMK, Feng M, Xiao J, Huang XR, Li P, et al. Novel IncRNA Erbb4-IR promotes diabetic kidney injury in $\mathrm{db} / \mathrm{db}$ mice by targeting miR-29b. Diabetes 2018; 67:731-44.

[6] Sathishkumar C, Prabu P, Mohan V, Balasubramanyam M. Linking a role of IncRNAs (long non-coding RNAs) with insulin resistance, accelerated senescence, and inflammation in patients with type 2 diabetes. Hum Genomics 2018; 12:41.

[7] Ge X, Xu B, Xu W, Xia L, Xu Z, Shen L, et al. Long noncoding RNA GAS5 inhibits cell proliferation and fibrosis in diabetic nephropathy by sponging miR-221 and modulating SIRT1 expression. Aging 2019; 11:8745-59.

[8] Kato M, Wang M, Chen Z, Bhatt K, Oh HJ, Lanting L, et al. An endoplasmic reticulum stress-regulated IncRNA hosting a microRNA megacluster induces early features of diabetic nephropathy. Nat Commun 2016; 7:12864.

[9] Hu M, Wang R, Li X, Fan M, Lin J, Zhen J, et al. LncRNA MALAT1 is dysregulated in diabetic nephropathy and involved in high glucose-induced podocyte injury via its interplay with $\beta$-catenin. J Cell Mol Med 2017; 21:2732-47

[10] Long J, Badal SS, Ye Z, Wang Y, Ayanga BA, Galvan $\mathrm{DL}$, et al. Long noncoding RNA Tug1 regulates mitochondrial bioenergetics in diabetic nephropathy. J Clin Invest 2016; 126:4205-18.

[11] Deng Q, Wen R, Liu S, Chen X, Song S, Li X, et al. Increased long noncoding RNA maternally expressed gene 3 contributes to podocyte injury induced by high glucose through regulation of mitochondrial fission. Cell Death Dis 2020; $11: 814$

[12] Zhao Y, Sun L, Wang RR, Hu JF, Cui J. The effects of mitochondria-associated long noncoding RNAs in cancer mitochondria: New players in an old arena. Crit Rev Oncol Hematol 2018; 131:76-82.

[13] Leucci E, Vendramin R, Spinazzi M, Laurette P, Fiers M, Wouters $\mathrm{J}$, et al. Melanoma addiction to the long non-coding RNA SAMMSON. Nature 2016; 531:518-22.

[14] Mercer TR, Neph S, Dinger ME, Crawford J, Smith MA, Shearwood $\mathrm{AM}$, et al. The human mitochondrial transcriptome. Cell 2011; 146:645-58

[15] Gusic M, Prokisch H. ncRNAs: New players in mitochondrial 
health and disease? Front Genet 2020; 11:95.

[16] Vendramin R, Marine JC, Leucci E. Non-coding RNAs: The dark side of nuclear-mitochondrial communication. EMBO J 2017; 36:1123-33.

[17] Stein CS, Jadiya P, Zhang X, McLendon JM, Abouassaly GM, Witmer $\mathrm{NH}$, et al. Mitoregulin: A IncRNA-encoded microprotein that supports mitochondrial supercomplexes and respiratory efficiency. Cell Rep 2018; 23:3710-3720.e3718.

[18] Chugunova A, Loseva E, Mazin P, Mitina A, Navalayeu T,
Bilan D, et al. LINC00116 codes for a mitochondrial peptide linking respiration and lipid metabolism. Proc Natl Acad Sci U S A 2019; 116:4940-5.

[19] Lewandowski JP, Dumbović G, Watson AR, Hwang T, JacobsPalmer E, Chang N, et al. The Tug1 IncRNA locus is essential for male fertility. Genome Biol 2020; 21:237.

[20] Calvo SE, Clauser KR, Mootha VK. MitoCarta2.0: An updated inventory of mammalian mitochondrial proteins. NucleicAcids Res 2016; 44:D1251-7. 